\title{
ABB 800xA applications in Process Control of a Stirred Reactor
}

\author{
Amrutha H, Rupesh K C
}

\begin{abstract}
The ABB industrial extended automation system $800 x A$ gives us to access and organize all plant information in a single application. The compact control builder is required to control a stirred reactor with the ability to process several liquid raw materials. This paper provides a functional design specification for designing an industrial stirred reactor and programming the AC 800M controller. The 800xA system gives a reliable, secure and control environment with all the built-in security features. The control process of the stirred reactor is simulated using $A B B$ 800xA simulation software.
\end{abstract}

Keywords-Control builder, Stirred reactor, AC 800M controller, 800xA system.

\section{INTRODUCTION}

The industrial system $800 x$ a automation is a more or less a comprehensive automation system. This 800xa system wraps the configuration and operation of both batch control and continuous control applications. The 800xa system has been evolved incorporating the information science along with its participation over the decades of victorious deliveries and with customer installation [1]. The system 800xa provides us a traditional system of control. This gives us to access all plant in information and to organize in single application. As expected, we have the functions like faceplates and graphics, alarm management and trending available. System 800xA uses quality hardware, protocols and operating system software's. This not only allows the data to be from ABB systems but from a different kind of sources such as other brands of control systems or from lab entries [2]. Raw material is fed into the reactor and the quantity is measured by flow meter. The temperature of the liquid is controlled with on off control using a heater and a cooler.

\section{SYSTEM 800XA ARCHITECTURE}

The System 800xA architecture deduce a system of devices and computers which communicate with one other over a dissimilar types of communication network, as embellished in the system shown here. The Workplaces uses either dedicated client computers or combined client/server machines allowing both client and server applications to run in one PC for larger configuration. The 800xa system

Revised Manuscript Received on July 22, 2019.

Amrutha H, PG student Dept. of EEE, Siddaganga Institute of Technology, Tumakuru, Karnataka. amrutha.amber567@gmail.com

Rupesh K C, Assistant Professor Dept. of EEE, Siddaganga Institute of Technology, Tumakuru, Karnataka. rupeshkc.sit@gmail.com communication is mainly based on the ethernet and IP/TCP networks. The workplaces usually run software's which provide different forms of user interaction whereas the server run software's which provide functionality to the system.

Systems which are small can run without a Domain Controller. In this case the nodes and users are handled by a Windows Workgroup. The configuration of users and security must then be done on all nodes separately within the Workgroup. The user handling in a Windows Domain is done from a central location - the Domain Server. Every domain must have at least one domain server, but for resilience a domain should have multiple domain servers. The Aspect Server provides the Aspect directory and the service related to the names, object management, security, etc. It is the heart of the $800 \mathrm{xA}$ system. As such it must be accessible to all nodes all of the time. The figure below shows the architecture of an 800xa system. In small systems the Aspect Server can be combined with other functions such as Application or Connectivity Servers in a single node (computer).

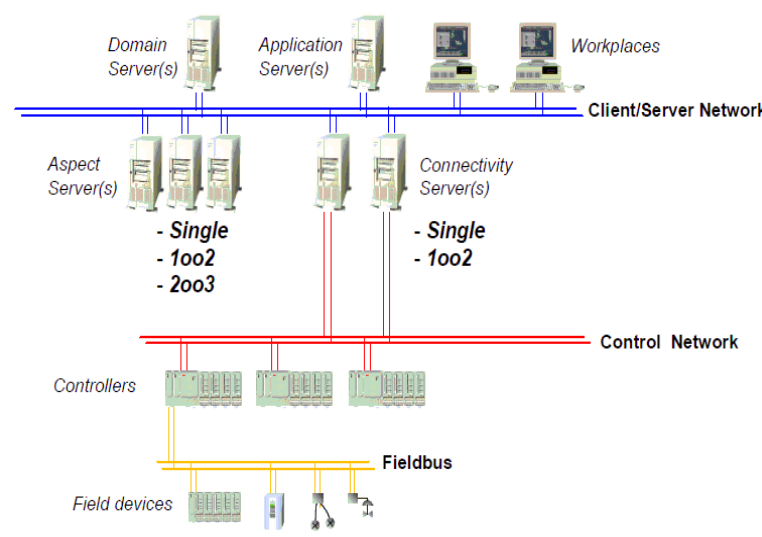

Figure 1: System 800xa Architecture

The Aspect Server supports "1 out of 2" or " 2 out of 3 " redundancy. In "1 out of 2" redundancy, the Aspect Directory is readable and writable as long as one Aspect Server is on-line. With "2 out of 3" redundancy, two Aspect Servers must be running to write to the Aspect Directory, but with any one Aspect Server online, the system is useable for operation but not for configuration. The Connectivity Server provides entrance to the device and also to the other particular sources throughout the network. There may be many groups of connectivity servers which exists in a system, each one of it serves one set of the data sources. The AC $800 \mathrm{M}$ Connectivity Server is one of many different types of Connectivity Servers in the 800xA System. 


\section{AC 800M CONTROLLER HARDWARE}

The $800 \mathrm{M}$ controller behaves either as a standalone controller or as a controller exploring local control actions in a control area which consists of many inter-connected controllers, servers and also operator servers when it is inserted with the desired control software. The AC800M controller can be equipped with various inputs/outputs systems which are either connected directly i.e. S800 input/output or through foundation field bus or profibus. The $800 \mathrm{M}$ controller is delivered to the customer without the control software. To supply the controller with the desired software, first we need to load the firmware and then we need to create the applications separately with the help of compact controller builder M software tool.

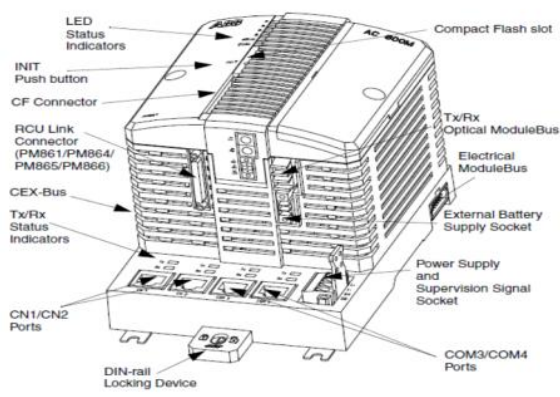

Figure 2: AC 800M hardware

The AC 800M Controller is composed of selection of parts which are mounted on the DIN rails horizontally which are housed within an enclosure. Many numbers of units composed of removable cover which is attached with screws and a base mounting plate. All the majority of the connections to the processor unit, communication interfaces, power supplies and also the connections to the systems and external buses is through the base plate which is encountered on the DIN rail. The figure 3.2 depicts the hardware structure of an AC 800M controller. This hardware appearance will not be applied to PM891.

The system AC $800 \mathrm{M}$ regulator provides us a low maintenance and cost-effective suspension for applications ranging from advanced distributed control systems to the small programmable logic controller, high level integrity system controller applications and combines control system. It is mainly viable to run both SIL and non SIL classified applications in the AC $800 \mathrm{M}$ high level integrity controller. This controller consists of PM810, SM811, PM865 and a high version of integrity control software and also feasible in redundant configuration. In case of SIL classified application the controller requires the use of SIL certified application then the standard S800 input/output parts can be equipped with AC $800 \mathrm{M}$ HI controllers. The below figure 3.3 epics the PM861 processor part which is also a part of the $800 \mathrm{M}$ controller. The processor part is dissimilar from PM891.
The central processing unit board consists of the ram memory and the microprocessor, the controllers which are equipped within the communication interface LED specifiers, real time clocks, INIT pump buttons and also a compact interface flash. The main actions of the supply board are to make the isolated circuits proof $+3.3 \mathrm{v}$ and $+5 \mathrm{v}$ supply to the input/output parts and to the central processing unit.

This board furthermore contains opto-isolated RS-232C receivers or drives together with a battery backup holder for real or memory time clock for service port. The cessation board, accommodated in the TP830 baseplate, is the place where $\mathrm{n}$ number of external connections terminates. Using the metallic equipment's of the housing the board is grounded into the DIN rail. The cessation board is equipped with screw terminals for redundant power supply monitoring and power supply with RJ45 connectors to that of the control network and also serial ports, the electrical module bus, the connector for those of the service ports and CEX-bus. The TP830 baseplate powers all those units in the CEX-bus and also the electrical module bus which is connected through $24 \mathrm{v}$ dc supply. It is feasible to connect S800 input/output clusters directly into the electrical built-in module bus which is situated on the right side of the baseplate in single CPU configuration. A processor part has a communication bus connector expansion which is situated on the left side of the baseplate. The CEX-bus furnishes for expanding the on-board ports with the extra communication interface.

\section{MATHEMATICAL MODEL OF STIRRED REACTOR}

To explore how the chemical process changes the behaviour with time under the impact of changes in the manipulated variables and in the disturbances and consequently to design an appropriate controller, we can employ two different methods [5]. In case of the chemical process, the physical equipment is made available to us. Consequently, we change deliberately the values of various inputs (disturbances, manipulated variables) and through appropriate measuring devices we observe. How the outputs (temperature, pressure, concentration) change with time in the chemical process. These kinds of procedures are time and effort consuming and it's therefore quite costly because, a large number of such experiments need be performed. 


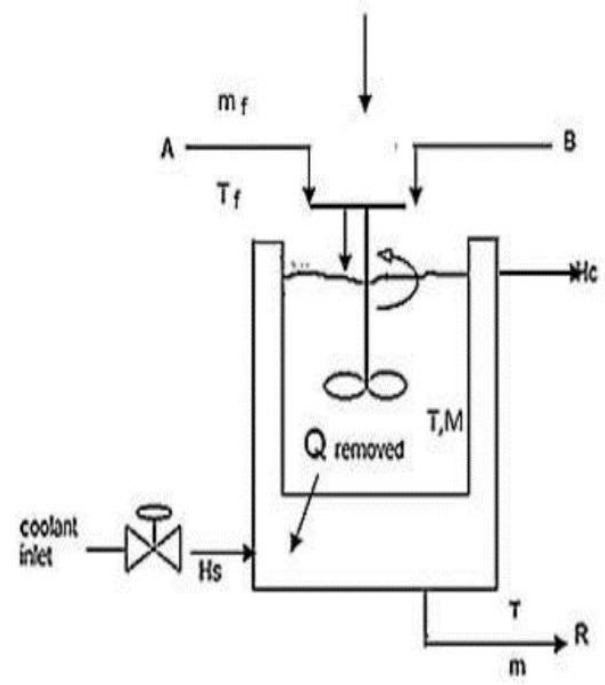

Figure 3: Model of Stirred Reactor

It is quite usual that we have to design the control system for a chemical process before the process plant has been constructed. In such a case we cannot rely on the experimental procedures, and we need a different way of representation of the chemical process in order to study its dynamic behaviour. This representation is mainly shown in terms of mathematical equations (algebraic, differential) whose solution gives the dynamic or static behaviour of the chemical process that we examine. A mathematical model usually describes a system by a set of variables and by a set of equations which establish the relationships between the variables. The values of the variables can be for example, practically real or integer numbers, Boolean values or strings. The variables represent some properties of the system e.g. measured system outputs often in the form of signals, counters, timing data, event occurrence (yes/no). The actual model is the set of functions that describe the relations between the different variables.

\section{SIMULATION AND EXPERIMENTAL RESULTS}

The Project Explorer in Control Builder $\mathrm{M}$ and the Plant Explorer / Engineering Workspace should be considered as two separate interfaces for building and maintaining control projects. As an engineer we will continuously shift between the Project Explorer (Control Builder M) and the Plant Explorer (800xA workplace) while building Control Projects.
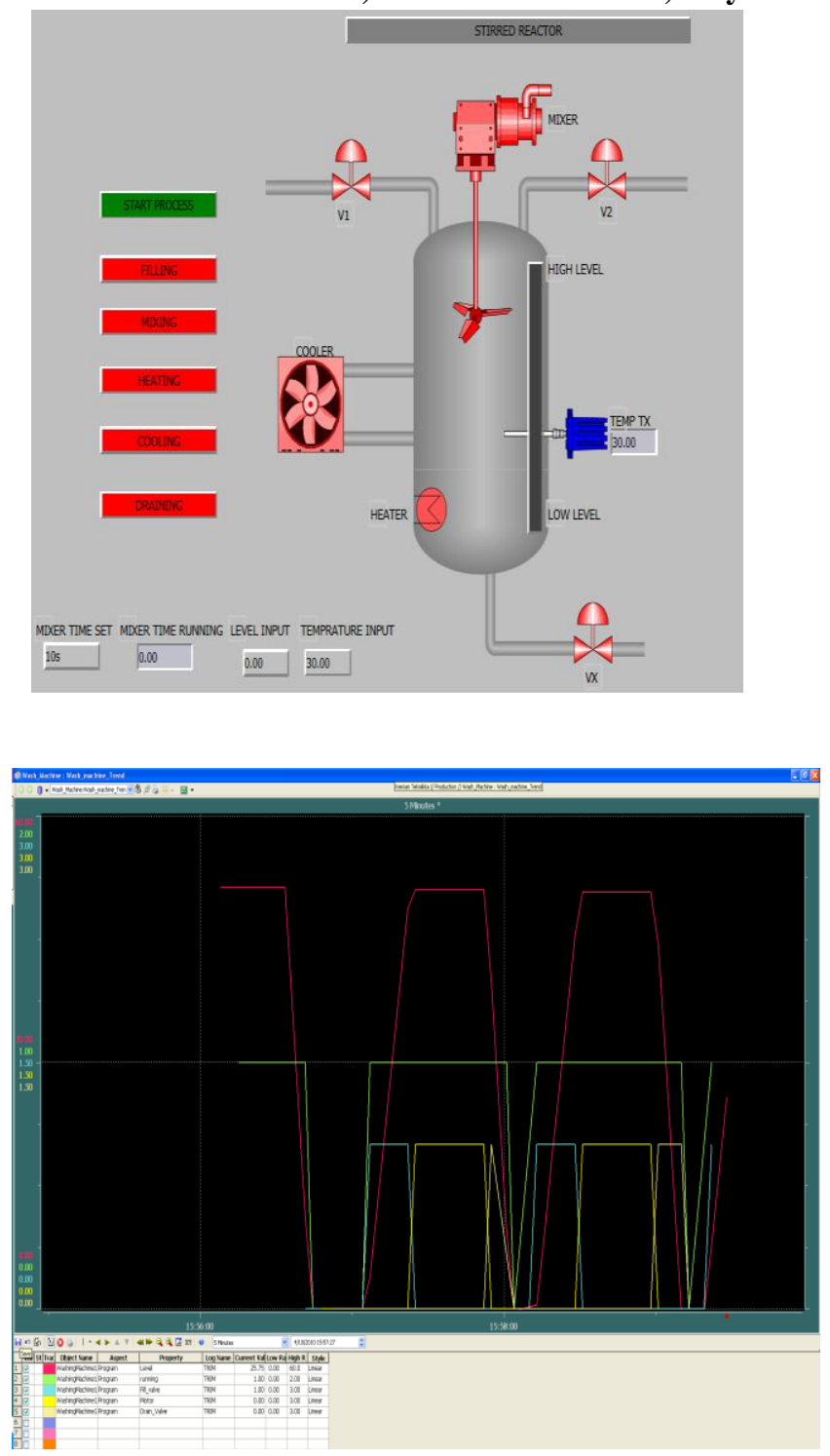

Figure 4: Simulation model and trend display of stirred reactor

The figure above shows the graphic display of a stirred reactor. The compact control builder is required to control a stirred reactor with the ability to process several liquid raw materials. The cooler is supplied with cooling water through the pump P1 to cool down the vessel temperature. The agitator is variable speed controlled. One digital output is used to start the motor. The speed of the motor may be controlled by a $0-20 \mathrm{~mA}$ reference signal.

After operating the reactor with fully automated sequence, the vessel is filled with certain quantity of raw materials. After completing filling, the content is heated up to the required temperature and then the mixing process starts and stops when the draining is completed. Programming the reactor to required design it keeps the process continues.

\section{CONCLUSION}

The 800xA system provides a reliable, secure and control environment with all the built in security features this enables the process to be in continues mode of operation

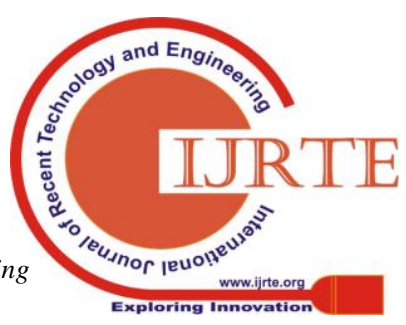


according the desired data. The process control of the stirred reactor is simulated using $\mathrm{ABB}$ 800xA simulation software which makes the process easy and provides efficient utilization of the material.

\section{REFERENCES}

[1] Introduction to system 800xA-3BUS095072

[2] System 800xA operations \& engineering workplace configuration-3BSE030322

[3] System 800xA control AC 800M configuration- BSE035980

[4] Smith, R.M (2005) Chemical Process: Design and Integration, John Wiley \& Sons, chichester.

[5] Houfar , F: Salah shoor, K. (2008). Adaptive control of CSTR using Feedback Linearization Based on Grey - Box Modeling, proceeding of FEEE International confe.

[6] Adaptive control systems : techniques and applications by V.V. chalam : $34-98$

[7] Srinivas Palanki, Soimitri Kolavennu, (2003). Simulation of control of CSTR process int. J. Engng Ed. Vol. 19 No 3, PP 398 